\title{
Caracterización textural de los músculos del tren delantero y propuesta de nuevos cortes en canales bovinas tres, cuatro y cinco estrellas procedentes del Urabá antioqueño (Colombia)
}

\author{
Diego A. Restrepo M ${ }^{1}$, Verónica Alzate A $^{\text {* }}$ y Kenneth R. Cabrera T² \\ (1) Universidad Nacional de Colombia, sede Medellín. Facultad de Ciencias Agrarias. Departamento de ingeniería \\ agrícola y de alimentos. Grupo de investigación en Ciencia y Tecnología de Alimentos (GICTA). Cra 65 a \# 59 a-110. \\ Medellín, Colombia. (correo-e: darestre@unal.edu.co; valzatea@unal.edu.co) \\ (2) Universidad Nacional de Colombia, sede Medellín. Facultad de Ciencias. Escuela de geociencias. Grupo de \\ investigación en Ciencia y Tecnología de Alimentos (GICTA). Cra 65 a \# 59 a-110. Medellín, Colombia \\ (correo-e: krcabrer@unal.edu.co)
}

${ }^{*}$ Autor a quien debe ser enviada la correspondencia.

Recibido Mar. 31, 2021; Aceptado Jun. 3, 2021; Versión final Jun. 30, 2021, Publicado Dic. 2021

\begin{abstract}
Resumen
El principal objetivo de esta investigación fue caracterizar texturalmente los músculos del tren delantero de bovinos del Urabá antioqueño en Colombia. Se diseccionaron 12 cuartos de canal clasificados en tres, cuatro y cinco estrellas según NTC 4271 (1996) y se separaron 28 de los músculos más voluminosos. Se determinó la dureza mediante una sonda Warner Bratzler acoplada a un texturómetro TA.XT System Plus. A partir de estos valores y usando los criterios de optimalidad (HG: max; HC: min; ASW: max) se buscó definir un bajo número de cortes primarios que presentaran terneza semejante, ofrecieran continuidad y, en lo posible, tuvieran mojones esqueléticos identificables, que permitieran establecer un protocolo de obtención rígido. Se concluye que el cumplimiento simultáneo de estas condiciones no es posible y que el sistema de clasificación por estrellas no permite establecer diferencias entre los valores de terneza por clasificación.
\end{abstract}

\section{Forehead muscle texture characterization and new cuts proposal for three, four, and five star bovine carcasses from the Urabá region in Antioquia (Colombia)}

\begin{abstract}
The primary aim of this research study was to characterize cattle forebrain muscle texture from Urabá, Antioquia (Colombia). Twelve carcass quarters classified as three, four, and five stars according to NTC 4271 (1996) were dissected and 28 of the most voluminous muscles were separated. Hardness was determined by using a Warner Bratzler probe coupled to a TA. XT System Plus texture analyzer. These values combined with optimal criteria (HG: max; HC: min; ASW: max) were used to identify a low number of primary cuts that were similar in tenderness while having continuity and identifiable skeletal landmarks that would allow establishing a rigid collection protocol. It was concluded that simultaneous fulfillment of these conditions was not possible and that a star classification system did not allow establishing differences between tenderness classification values.
\end{abstract}

Keywords: bovine cuts; muscle fiber; tenderness; cattle forebrain; classification 


\section{INTRODUCCIÓN}

Garantizar una correcta comercialización de los cortes del tren delantero de las canales bovinas basados en evidencias de su calidad textural, presenta una gran oportunidad para generar valor agregado, esto debido principalmente a que en Colombia actualmente existe una carencia en la estandarización de estos cortes dejando a voluntad del carnicero la forma de realizarlos y comercializarlos. La textura es uno de los atributos sensoriales de mayor importancia para los consumidores, y esta se define como el conjunto de propiedades reológicas percibidas por los sentidos, como por ejemplo la dureza, la cual se define como la cantidad de fuerza máxima requerida para comprimir el alimento entre los molares o la lengua y el paladar (González et al., 2015) revelando así la calidad del producto al momento del consumo. Esta característica en la carne está influenciada por tres factores como, el efecto acto miosina (aportado por las fibras musculares), el efecto de fondo (aportado por el tejido conectivo), y la densidad aparente o efecto lubricante (aportado por la grasa intramuscular) (Chun et al., 2020). A través de los años se ha demostrado que la terneza está asociada a factores específicos del músculo como el contenido de grasa, de colágeno, el sistema de actividad proteolítica, la localización y función del músculo y, el tipo de fibra muscular. (Wright et al., 2018; Yeh et al, 2018)

Los cortes o carnes del tren delantero del bovino son considerados de calidad segunda e industriales, precisamente porque esta especie realiza la mayoría de la fuerza para su desplazamiento con esta parte del cuerpo, ejercitándolo mucho más que el posterior, y en razón del sistema productivo usado principalmente en la región del Urabá, extensivo o pastoreo, los animales cuando se sacrifican exhiben estas carnes como más duras; sin embargo, el USDA (United States Department of Agriculture) en el año 2107 presentó a los consumidores el músculo Subescapularis como un novedoso corte, al igual que la parte caudal del músculo Infraespinatus, quienes exhibían un valor agregado por su terneza (Yeh et al ., 2018), por lo que, se abre camino a la posibilidad de poder destacar el potencial que pueden tener los cortes del tren delantero.

Della Rosa et al., (2017), atribuyen la terneza al tipo de fibra, además de indicar que el tipo de metabolismo de la fibra muscular también afecta el contenido de enzimas proteolíticas en las que las fibras oxidativas presentan mayor concentración de calpaínas que las fibras glucolíticas. Motter et al., (2018) en estudios realizados en tres músculos de características contrapuestas como el infraespinatus, supraespinatus y tricpes brachii, el Infraespinatus y triceps brachii evidenciaron mayor proporción de fibras tipo I y IIA (músculos oxidativos) exhibiéndose como los más tiernos, mientras que en el músculo Semitendinosus predominó el tipo de fibra IIX (músculos glucolíticos), comportándose como el más duro. Debido a que los cortes comerciales están compuestos por paquetes musculares, los cuales difieren entre sí en su metabolismo o propiedades bioquímicas (glucolítico u oxidativo) y en su ubicación dentro de la canal, es muy factible que dentro de un mismo corte se presenten ternezas diferentes, ya que cada músculo va a responder de una manera diferente al proceso de conversión del músculo en carne (Nair et al., 2019).

Por lo tanto, caracterizar los principales músculos del tren delantero, y explicar su terneza con base en los tipos de fibras musculares y su metabolismo, puede aclarar las diferencias en la calidad de los mismos y de esta manera proponer nuevos cortes (Clusters de músculos) según su terneza y localización al interior de la canal, brindando así la oportunidad de dar un valor agregado y evitar la subutilización de estos. Con la realización de estudios de perfiles texturales en la carne de bovino, la industria cárnica tendría la posibilidad de comercializar cortes constituidos por músculos individuales o grupos de músculos basados en características fisicoquímicas y rasgos de calidad (Nair et al., 2019). El objetivo del presente estudio es caracterizar texturalmente los principales músculos del tren delantero de canales bovinas clasificadas en tres, cuatro y cinco estrellas procedentes del Urabá Antioqueño (en Colombia), buscando el establecimiento de nuevos cortes con valor agregado que garanticen al consumidor una uniformidad en la terneza del producto.

\section{METODOLOGÍA}

La presente investigación se realizó usando 12 cuartos delanteros obtenidos mediante el corte semipistola de ganado bovino cebuino, machos, castrados, procedentes del noroccidente de Colombia (Departamento de Antioquia, subregión del Urabá), después de faenarlas siguiendo los lineamientos definidos por el Ministerio de Salud y de la Protección Social de Colombia, en las instalaciones del frigorífico Central Ganadera de Medellín (Colombia), en la planta de desposte de Supermercados EURO Quality Beef (Itagüí, Colombia), y en los laboratorios de Productos Cárnicos y de Control y Calidad de Alimentos de la Universidad Nacional de Colombia, sede Medellín.

\section{Clasificación de las canales}

La clasificación de las canales se llevó a cabo según los parámetros establecidos por el manual de clasificación de canales bovinas del ICTA que considera edad, grado de acabado, conformación de la canal, condición sexual, longitud de la canal y rendimiento (NTC 4271,1996). 


\section{Delimitación anatómica de los músculos del tren delantero}

Para esta etapa se eligieron 6 canales (12 cuartos delanteros) clasificadas como tres, cuatro y cinco estrellas de acuerdo con el sistema ICTA, las cuales, después de refrigeradas en el frigorífico, fueron diseccionadas músculo a músculo según el Atlas de Anatomía Topográfica de los Animales Domésticos (Popesko,1998). De allí se identificaron 28 músculos importantes (por su tamaño) que fueron adecuadamente rotulados y empacados, para ser llevados a las instalaciones de los laboratorios de Productos Cárnicos y Control de Calidad de Alimentos de la Universidad Nacional de Colombia, sede Medellín, en neveras portátiles que permitieran mantener la cadena de frío. Para el análisis fueron acondicionados al rojo con la ayuda de un cuchillo, divididos en tres secciones (dos extremos y un medio), y ultra-congelados mediante el equipo Tecnomac, modelo E10-35 A"G", para después ser dispuestos para su análisis. Los músculos obtenidos se indican en la tabla 1 con su respectivo código de identificación

Tabla 1. Identificación de músculos del tren delantero de una canal bovina

\begin{tabular}{|c|c|}
\hline Identificación & Músculo \\
\hline$B B$ & Biceps brachii \\
\hline$B C$ & Brachiocephalicus \\
\hline$C L$ & Caput laterale Tricipitis brachii \\
\hline CLO & Caput longum Tricipitis brachii \\
\hline$C M$ & Caput medium Tricipitis brachii \\
\hline$C A$ & Cutaneus abdominis \\
\hline$D E$ & Deltoideus \\
\hline$D F$ & Diaphragma \\
\hline$E X$ & Extensorum \\
\hline$F L$ & Flexorum \\
\hline IN & Infraspinatus \\
\hline$L D$ & Latissimus dorsi \\
\hline$L C$ & Longissimus cervicis \\
\hline LDS & Longissimus dorsi \\
\hline$L L$ & Longissimus lumborum \\
\hline$O A E$ & Obliquus externus abdominis \\
\hline$O M$ & Omotransversarius \\
\hline$P C$ & Pars cervicalis trapezii \\
\hline$P A$ & Pectoralis ascendens \\
\hline$P D$ & Pectoralis descendens \\
\hline$R A$ & Rectus abdominis \\
\hline$R M$ & Rhomboideus \\
\hline$S C$ & Serratus cervicalis \\
\hline$S T$ & Serratus thoracis \\
\hline$S P$ & Splenius \\
\hline$S C P$ & Sterno-cephalicus \\
\hline$S U$ & Supraspinatus \\
\hline$T T$ & Transversus thoracis \\
\hline
\end{tabular}

\section{Evaluación del pH}

El pH se evaluó durante el tiempo de almacenamiento en refrigeración, siguiendo la metodología de medición directa en carne fresca descrita por (Braña et al., 2011), utilizando un pHmetro HANNA instruments pH 211, equipado con electrodo de penetración y sonda de temperatura. Dichas mediciones se efectuaron en cada uno de los músculos crudos las primeras 24-48 horas post mortem, realizando una pequeña incisión sobre el músculo e introduciendo el electrodo en él hasta que el valor del pH se estabilizó.

\section{Evaluación de la terneza}

El análisis de medida por fuerza de compresión fue realizado según las directrices de (Bourne, 1978), usando un TAXT System Plus (England), el cual se basa en la ruptura de la muestra con una sonda Warner Bratzler. Las muestras para evaluar se cocinaron en un horno MKN, Central Eficiente de Cocción GmbH \& Co, hasta alcanzar una temperatura interna de $72^{\circ} \mathrm{C}$, luego se enfriaron a $4^{\circ} \mathrm{C}$ y posteriormente se cortaron en forma de paralelepípedo con un área de $1 \mathrm{~cm} \times 1 \mathrm{~cm}$ y $5-6 \mathrm{~cm}$ de largo para ser analizadas en el texturómetro. Se 
realizaron 6 repeticiones por músculo utilizando las siguientes especificaciones: Velocidad pre-ensayo: 2,00 $\mathrm{mm} / \mathrm{s}$, velocidad ensayo: $2,00 \mathrm{~mm} / \mathrm{s}$, velocidad post ensayo: $10,00 \mathrm{~mm} / \mathrm{s}$, con una distancia de $40,0 \mathrm{~mm}$ y una celda de $50 \mathrm{Kg}$.

\section{Propuesta de cortes}

Para establecer la propuesta inicial de cortes usando la terneza como criterio básico de agrupamiento, se adoptaron los siguientes criterios de optimalidad estadística: HG (máx) Mide la capacidad de la clusterización de reproducir las distancias en órdenes de magnitud. Usa la correlación no lineal gamma de Goodman y Kruskal. HC (min) Brecha entre la partición propuesta y la mejor partición jerárquica teórica posible con este número de grupos y con estas distancias (Hubert y Schultz, 1976). ASW (máx) Coherencias de la asignación. Alta coherencia indica significativas distancias de grupo a grupo y una fuerte coherencia al interior del grupo (Rousseeuw, 1987). Como segundo criterio complementario de conformación de cortes, se adoptó la vecindad.

El análisis de clúster tomó cada músculo como una unidad fundamental y se construyeron dos matrices de distancia, una para la terneza y otra para vecindad. Para la construcción de la primera se definió la distancia como la diferencia entre los valores de terneza de cada músculo, de tal manera que si dos músculos presentan una terneza similar la distancia es cercana a cero, por el contrario, si la diferencia es apreciable, la distancia entre los músculos es alta. En la otra matriz la distancia estuvo dada por la vecindad. En este caso se tomó una similitud definida como el porcentaje ya sea de participación de vecindad con el respectivo músculo o con sus vecinos. De tal forma que la vecindad se estableció por un lado del músculo con sus vecinos y de los vecinos con el propio músculo. Esta matriz se transformó en una matriz de distancia al restarle a uno (1) los valores de vecindad y promediar los porcentajes para obtener una matriz simétrica.

Las dos matrices se ponderan de acuerdo con criterio que se quiera representar que para el caso fue desde el 0:100 al 50:50 (relación terneza: vecindad). La matriz resultante final de distancia se utiliza para construir el clúster jerárquico respectivo. Por tratarse de unidades diferentes, se estandarizan para ser comparables. En la tabla 2 se presenta una fracción de la matriz base para la vecindad. Con los criterios de terneza y vecindad definidos, se corrió mediante el programa $R$ ( $R$ Core Team,2021), un análisis de clúster 0 agrupamiento en proporciones vecindad de ponderación de la terneza de 0:100 y 50:50, arrojando los grupos de músculos que conformarían las propuestas de cortes primarios.

Tabla 2. Fracción de la matriz de vecindad de los músculos del tren delantero de la canal bovina.

\begin{tabular}{|l|c|c|c|c|c|}
\hline \multicolumn{1}{|c|}{ Musculo } & Gran dorsal & Supraespinoso & Infraespinoso & Deltoides & $\ldots .$. \\
\hline Gran dorsal & 1 & 0,048 & 0,048 & 0,048 & \\
\hline Supraespinoso & 0,115 & 1 & 0,192 & 0,153 & \\
\hline Infraespinoso & 0,208 & 0,166 & 1 & 0,125 & \\
\hline Deltoides & 0,098 & 0,098 & 0,196 & 1 & \\
\hline \multicolumn{2}{c|}{$\ldots$} & & & & 1
\end{tabular}

\section{Análisis estadístico}

Para el análisis de las variables terneza y pH se utilizó un modelo mixto en el cual la unidad experimental es el animal y se registraron los valores promedios de cada variable. El modelo mixto se establece porque a cada músculo de un mismo animal se le hicieron varias mediciones. Y a cada animal, a su vez, se le evaluaron diferentes músculos. Se realizó la evaluación de los parámetros mediante una ANOVA para determinar diferencias significativas $(P<0,001)$ o no $(P>0,001)$; posteriormente por medio de la prueba de Tukey en modelo de efectos mixtos, se compararon los promedios para cada músculo en cada variable. (Searle et al.,1980)

\section{RESULTADOS Y DISCUSIÓN}

A continuación, se indican los resultados obtenidos para la terneza y el pH de los músculos del tren delantero de canales bovinas tres, cuatro y cinco estrellas y las dos propuestas de corte en base a la clusterización de los músculos según una proporción vecindad: terneza de 0:100 y 50:50.

\section{Evaluación de la terneza en músculos.}

En la figura 1 se indican los resultados de fuerza de corte para los 28 músculos evaluados en las tres clasificaciones. No se encontraron diferencias significativas para la terneza de los músculos entre canales clasificadas diferentemente, $(P>0,001)$ evidenciándose así que la clasificación no es un factor determinante para indicar de manera general la terneza de los músculos provenientes de estos animales. 


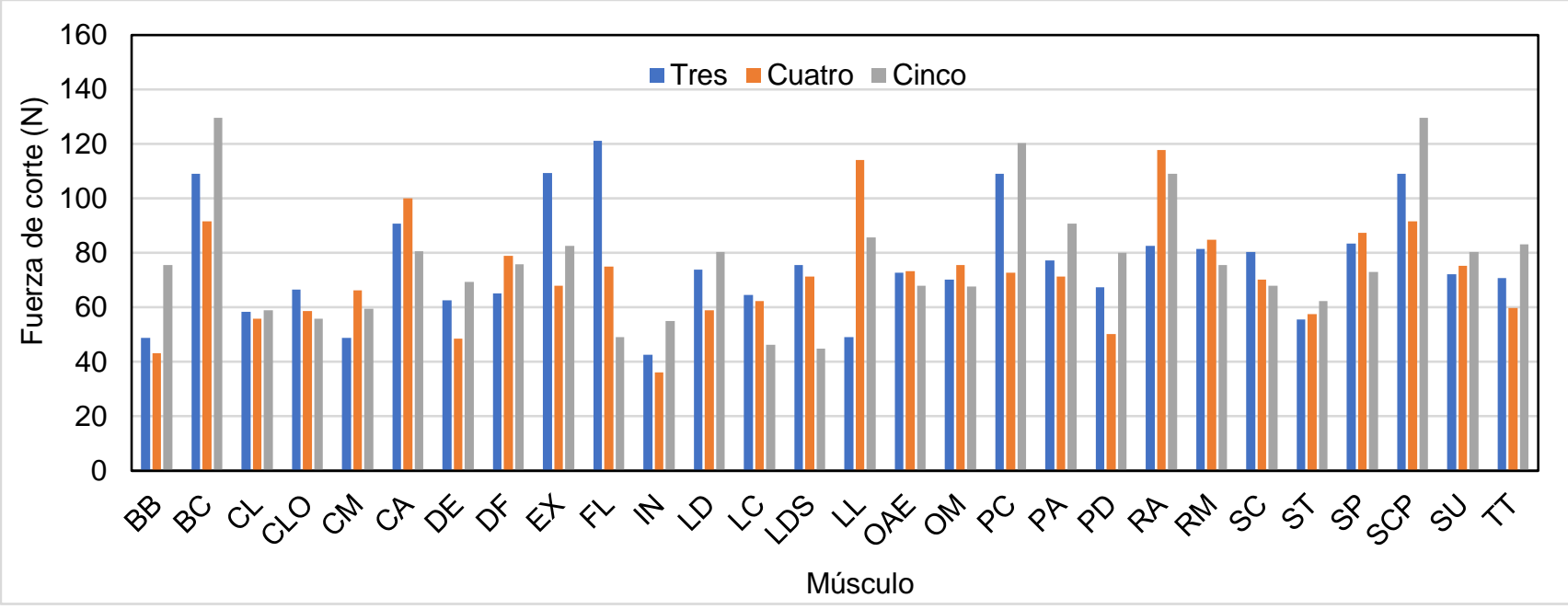

Fig 1. Valores de fuerza de corte promedio para los 28 músculos evaluados en las clasificaciones tres, cuatro y cinco estrellas

Aunque la edad del animal es uno de los factores principales para clasificar una canal, debido a la relevancia que puede tener en cuanto a la terneza, es importante tener en cuenta que el intervalo de edad entre cada categoría o clasificación no difiere significativamente (Entre un año y año y medio de diferencia entre clasificaciones), por lo tanto, es muy probable que otros factores tanto intrínsecos como extrínsecos influyan de manera más significativa en la terneza de determinado músculo, tal como lo indican (Nair et al., 2019), quienes expresan que las distintas características bioquímicas de los músculos son las que determinan en gran porcentaje la expresión de las proteasas extrínsecas, además del contenido de tejido conectivo y la longitud del sarcómero lo cual contribuye en la variación intermuscular de la terneza. En la figura 2 se observan los valores promedio de fuerza de corte de mayor a menor para los 28 músculos evaluados.

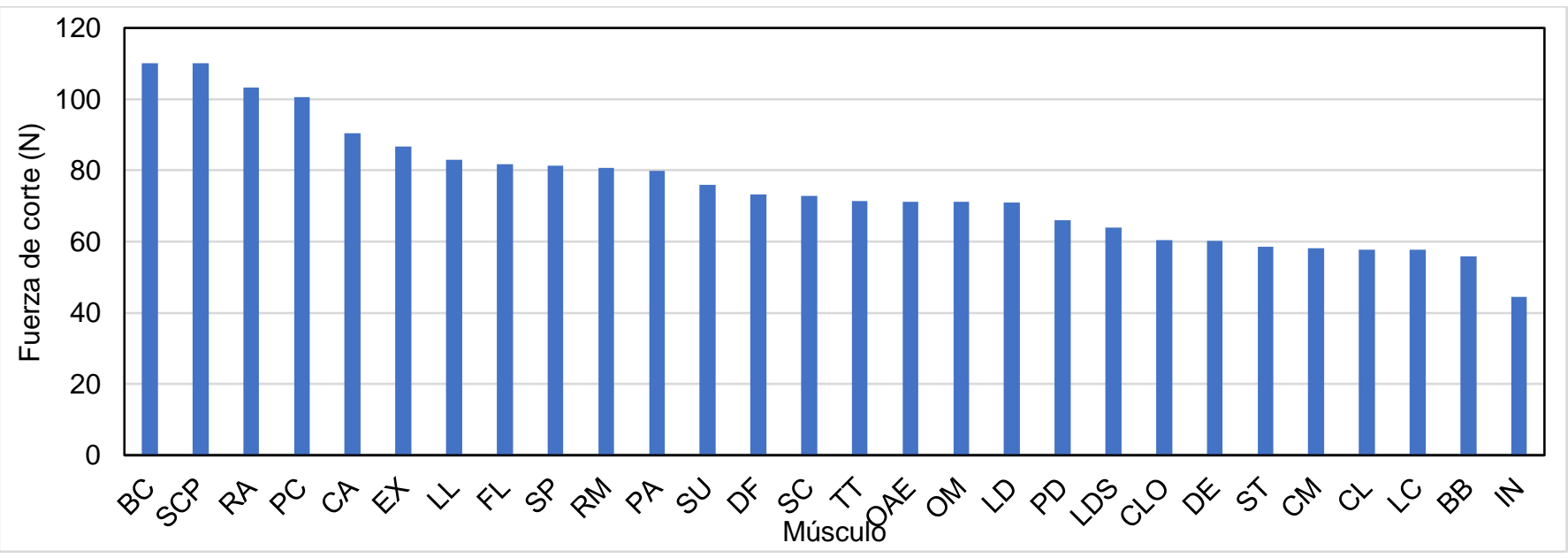

Fig 2. Fuerza de corte promedio en músculos del tren delantero

Se encontraron diferencias significativas $(P<0,001)$ para la fuerza de corte entre los músculos. Dentro de los principales factores que influyen en la terneza de un determinado músculo se encuentra el tipo de fibra, la cual puede presentar una gran variabilidad entre músculos diferentes, incluso dentro del mismo músculo. Este tipo de fibra va a depender principalmente de la función del músculo, donde aquellos músculos con mayor proporción de fibras tipo I (Oxidativos y de contracción lenta) como lo son el Biceps brachii e Infraspinatus (Motter et al., 2021), tienden a presentar una mayor terneza, debido principalmente a que las fibras son de menor diámetro, muy vascularizadas, y con poco glucógeno. Desde otra óptica, aquellos músculos con mayor proporción de fibras tipo II (Glucolíticos y de contracción rápida) como los son Longissimus thoracis, Rectus abdominis, semimembranosus, entre otros (Listrat et al., 2020) se consideran de menor terneza debido a que sus fibras son de mayor diámetro, presentan pocas mitocondrias, y están poco vascularizados, pero contienen mucho glucógeno.

Generalmente los músculos relacionados con el movimiento, como los de la pierna, brazo y cuello se esperaría que fueran más duros, mientras que los músculos del lomo y la costilla que están involucrados en el apoyo y la postura lo esperado es que tiendan a ser más tiernos (Nair et al., 2019). Sin embargo, en los 
resultados del presente estudio, algunos de los principales músculos que conforman el brazo (responsable del movimiento) presentaron menores fuerzas de corte (mayor terneza) en comparación con los demás músculos, inclusive a los posturales como el Longissimus dorsi. En el caso de los músculos Infraespinatus, Caput medium Tricipitis brachii y Biceps brachii estos fueron los que presentaron los menores valores de fuerza de corte en las canales evaluadas $(42,1 \mathrm{~N}, 55,5 \mathrm{~N}$ y 53,6 N respectivamente) considerándolos así, como unos de los músculos más tiernos. Estos resultados concuerdan con los obtenidos por (Motter et al.,2018), quienes realizaron sus trabajos en los músculos Infraespinatus, Semitendinosus y Triceps Brachii, observando diferencias principalmente en terneza y composición de tipo de fibra, donde el Infraespinatus fue catálogado como uno de los más tiernos, el semitendinosus entre los menos tiernos, y el triceps brachii en un músculo de terneza intermedia, encontrando que el músculo Infraespinatus mostró mayor porcentaje de fibras I y Ila, el Semitendinosus mostró una relación inversa, con mayor predominancia de fibras II y en el Triceps Brachii se identificó aproximadamente un tercio de cada fibra. En cuanto al músculo Longissimus Thoracis, (Costa et al., 2015), identificaron una mayor proporción de fibras tipo Il y una fuerza de corte de 77,1 N.

Los músculos Longissimus dorsi, Latissimus dorsi y Longissimus lumborum presentaron algunas diferencias entre sí. El Longissimus lumborum, fue el que presentó una mayor fuerza de corte $(83,1 \mathrm{~N})$, mientras que el Longissimus dorsi y el Latissimus dorsi presentaron valores intermedios (63,9N y 71,02N respectivamente), sin embargo los tres se clasifican como músculos de alta dureza, lo cual se asemeja a lo evidenciado por (Choi et al., 2010), quienes evaluaron el tipo de fibra en el músculo Longissimus dorsi y señalan una mayor proporción de fibras blancas (Tipo IIB) de mayor diámetro, más tejido conectivo y menos grasa intramuscular, igualmente (Nair et al.,2019), encontraron valores de fuerza de corte de 60,85 N para el músculo Longissimus lumborum, lo cual indica que a pesar de ser músculo responsable de la postura, los altos valores de fuerza de corte lo catalogan como duro. Esto indica que afirmaciones como las que expresan que los músculos de soporte son más tiernos que los involucrados en el movimiento son menos válidas cuando se evalúan una amplia gama de músculos individuales. Los músculos Brachiocephalicus y Sterno-cephalicus fueron los que presentaron la mayor fuerza de corte, de $135,1 \mathrm{~N}$ y $110,1 \mathrm{~N}$ respectivamente, lo cual se debe principalmente a su ubicación en la región del cuello, que es una parte del animal que está en constante movimiento, favoreciendo así su mayor dureza.

\section{Evaluación del pH en músculos}

La evaluación del pH se realizó iniciando con la medición a las 24 horas post mortem en el músculo Longissimus dorsi para tener un indicador general en el descenso adecuado del $\mathrm{pH}$ en las canales. En promedio el $\mathrm{pH}_{24}$ fue de 5,52, estando dentro del rango normal del $\mathrm{pH}$ en ese tiempo, el cual debe ser $<5,8$ (Apaoblaza et al., 2015). Posteriormente entre el día 3-4 post mortem se realizó la medida del pH a cada músculo simultáneo a la medición de la terneza. En la figura 3 se presentan los valores de pH de manera ascendente para cada músculo.

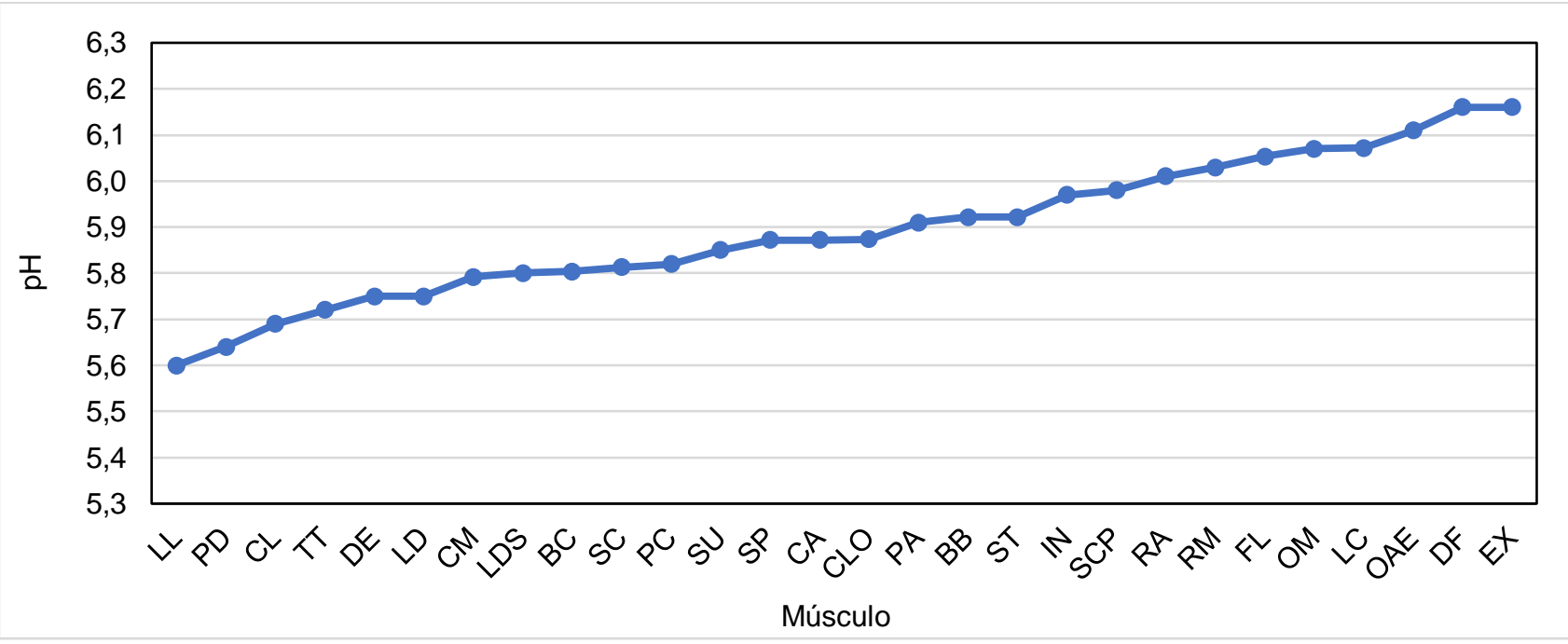

Fig 3. Valores promedio de pH para los principales músculos del tren delantero 3-4 días post mortem.

No se presentaron diferencias significativas $(P>0,001)$ para $\mathrm{pH}$ entre canales clasificadas diferentemente, pero si entre los músculos $(P<0,001)$. El potencial glucolítico del músculo del bovino representa una fuente importante en la variación de la calidad de la carne, ya que este va a definir las reservas de energía que el músculo tendrá al momento del sacrificio, lo cual está correlacionado con el pH final de la carne (Wright et al., 2018). Una mayor proporción de fibras glucolíticas va a aumentar el grado de disminución del pH en el periodo post mortem, lo cual puede afectar de manera significativa el desarrollo de la calidad de la carne. Los músculos 
con mayor proporción de fibras tipo I (Oxidativas), al presentar menor contenido de glucógeno exhiben valores de pH más altos, además de que este tipo de fibra es de menor tamaño, lo cual tendrá un efecto sobre la terneza del músculo. Anderson et al., (2012), al evaluar la terneza de los músculos que conforman el corte de la pierna, evidenciaron que el músculo Vastus intermedius presentó una menor fuerza de corte en comparación con los otros músculos, lo que se debió probablemente a los mayores valores de pH que obtuvo este músculo, lo cual se relaciona también con la mayor proporción de fibras tipo I. Además, el autor indica que los valores altos de $\mathrm{pH}$ pueden tener efecto en la actividad $\mu$-calpaína y por lo tanto podría influir en la proteólisis y sensibilidad posterior en ese músculo. Wu et al., (2014) concluyó que aquellos músculos con un $\mathrm{pH}$ final alto presentan una menor fuerza de corte, lo cual se relaciona con una mayor tasa de degradación de proteínas a estos valores de $\mathrm{pH}$.

Para el presente estudio se pueden correlacionar dichos resultados al considerar que algunos de los músculos que conforman el brazo (Infraespinatus, Bicepsbrachii, Tricepsbrachii y Caput medium Tricipitis brachii) podrían clasificarse como oxidativos, exhibiendo pH más elevados, de 5,8 en promedio, lo cual explicaría la menor fuerza de corte obtenida en los mismos. Datos similares fueron obtenidos por (Purchas et al., 2008); quienes evidenciaron un $\mathrm{pH}$ final en el músculo Infraespinatus de 5,73. Igualmente en el caso del músculo Longissimus dorsi, se podría considerar glucolítico al presentar una mayor fuerza de corte, correlacionado igualmente con sus menores valores de $\mathrm{pH}$ (5,6 en promedio) comparado con los músculos de la región del brazo, contrastando así estos valores con los encontrados por (Wu et al., 2014), quienes hallaron valores de $\mathrm{pH}$ de 5,6 para este mismo músculo, igualmente (Choi et al.,2010), indican valores de $\mathrm{pH}_{24}$ de 5,57. Es importante considerar que aparte del tipo de fibra y del potencial glucolítico u oxidativo del músculo, un factor determinante en la terneza de la carne es el sistema calpaína-calpastatina, donde la calpaína es la principal responsable de la proteólisis y la terneza y por su parte la calpastatina representa una actividad inhibidora a la calpaína. (Wright et al., 2018).

Varios estudios indican que la genética está relacionada directamente con la acción del sistema enzimático calpaína-calpastatina, y que esta última presenta una mayor actividad en el ganado Bos indicus, genotipo predominante en la producción ganadera de la región del Urabá Antioqueño, lo cual se traduce en una menor sensibilidad del músculo, es decir en una menor terneza en este grupo racial. (Wright et al., 2018), al evaluar la influencia de la genética Brahman sobre las propiedades de las fibras musculares, la degradación de las proteínas y la terneza, evidenciaron que la terneza de los músculos de aquellos grupos con genética Brahman se catalogaron como "Ligeramente duros", lo cual concuerda con lo obtenido en el presente estudio, donde todos los músculos evaluados según la escala de la Universidad de Nebraska se clasifican como de dureza intermedia o mayor (Sullivan y Calkins ,2007). Wright et al., (2018), indican que el grado de autolisis de la calpaína -1 a las 24h post mortem disminuyó a medida que el porcentaje de Brahman aumentaba, y por su lado el contenido de calpastatina aumentaba, lo cual soporta la menor terneza en este grupo racial.

\section{Propuesta de cortes}

En la figura 4 se observa la distribución de los criterios de optimalidad utilizados y los posibles números de grupos de músculos que conformarían la propuesta de cortes para una proporción vecindad: terneza de 0:100. Para definir el número de clusters que más se ajuste a la practicidad en el momento del desposte se verifica que los criterios cumplan las condiciones de los tres índices simultáneamente. Para el caso, se escoge el valor de diez, teniendo en cuenta que el criterio HG debe ser un valor máximo, el criterio HC un valor mínimo y el criterio ASW un valor máximo. Aunque si bien hay otros números de clusters en los cuales los criterios de optimalidad se cumplen, con base a la realidad del desposte un número mayor ya no sería tan viable.

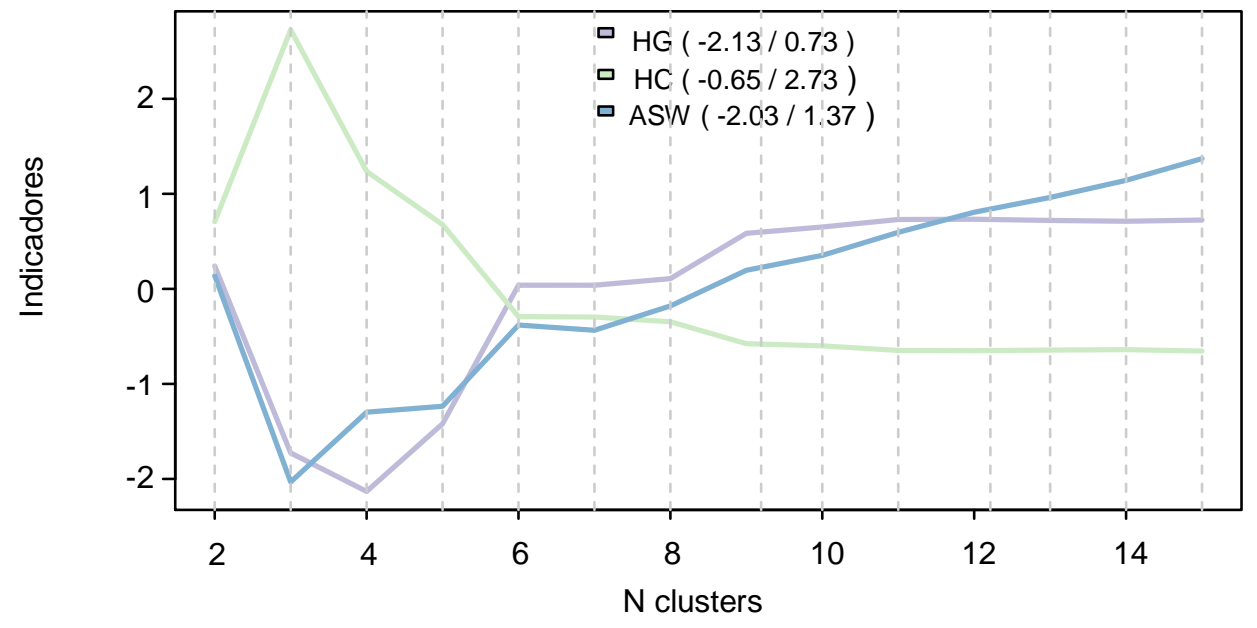

Fig. 4: Distribución de criterios de optimalidad para proporción 0:100 


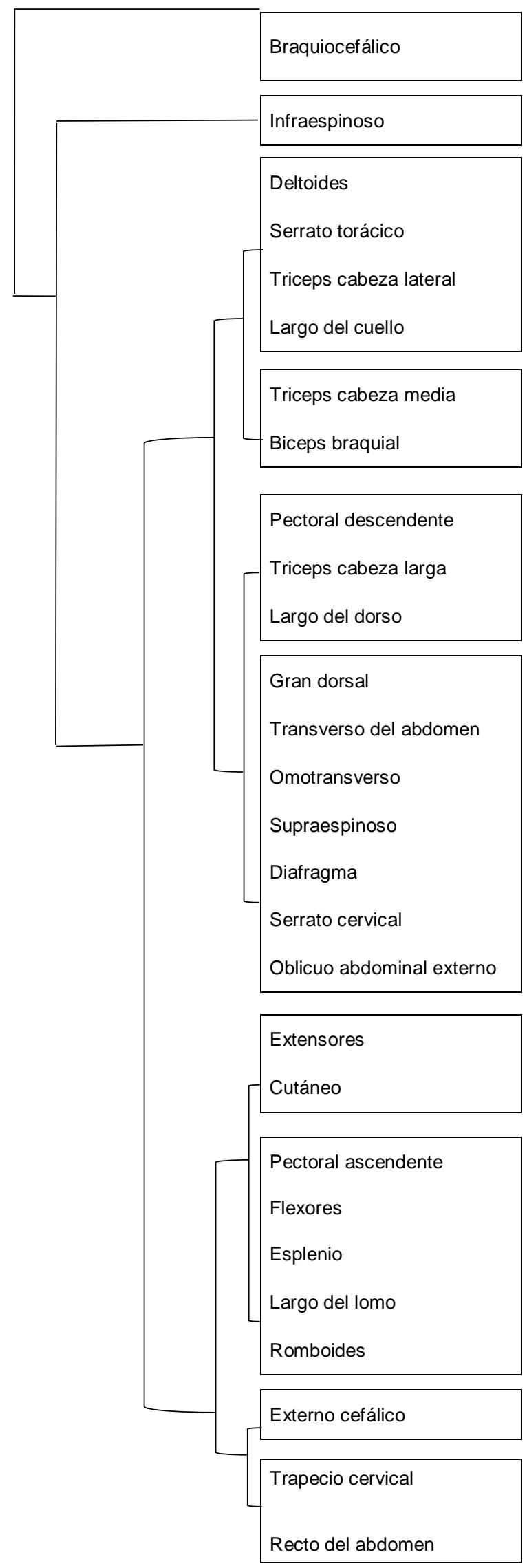

Fig. 5: Grupos de músculos según proporción vecindad: terneza 0:10 
Se propone realizar un primer acercamiento a la viabilidad de obtener cortes formados por músculos que presenten similar terneza sin tener en cuenta la ubicación o vecindad entre ellos. En la figura 5 se muestran los diez clusters o grupos de músculos a proponer para la proporción vecindad: terneza de 0:100. En la figura se proponen diez cortes compuestos por músculos de similar terneza sin tener en cuenta su ubicación anatómica o vecindad. Teniendo en cuenta que en la práctica habitual del desposte se procede a destazar los cortes ubicados en el brazo y antebrazo inicialmente, seguido de los que componen la porción cervical y finalmente los ubicados en el dorso - lomo y abdomen, esta propuesta es inviable debido a que son escasos los cortes que presentan continuidad, como por ejemplo el cluster 6 incluye dentro del mismo un músculo ubicado en la región dorsal, dos músculos de la región abdominal, dos músculos de la región cervical, un músculo de la región del brazo y un músculo de la parte interna de la canal como el diafragma, lo cual impide su implementación como único corte, generando así varios subcortes.

En general esta propuesta implementada en la práctica sería inviable ya que arrojaría en total 20 cortes primarios (número bastante elevado), lo cual es ilógico llevar a cabo por la inexistencia de mojones anatómicos claros, lo cual indica que estandarizar un corte únicamente en base a la terneza no es posible debido principalmente a la gran variación que puede presentar los músculos entre sí. Con base en los resultados obtenidos con la proporción vecindad: terneza 0:100, se propone evaluar la proporción 50:50, donde ya la terneza y la vecindad tendrán una participación por igual. En la figura 6 se observa la distribución de los criterios de optimalidad y los posibles grupos de músculos que conformarían la propuesta de cortes para esta proporción.

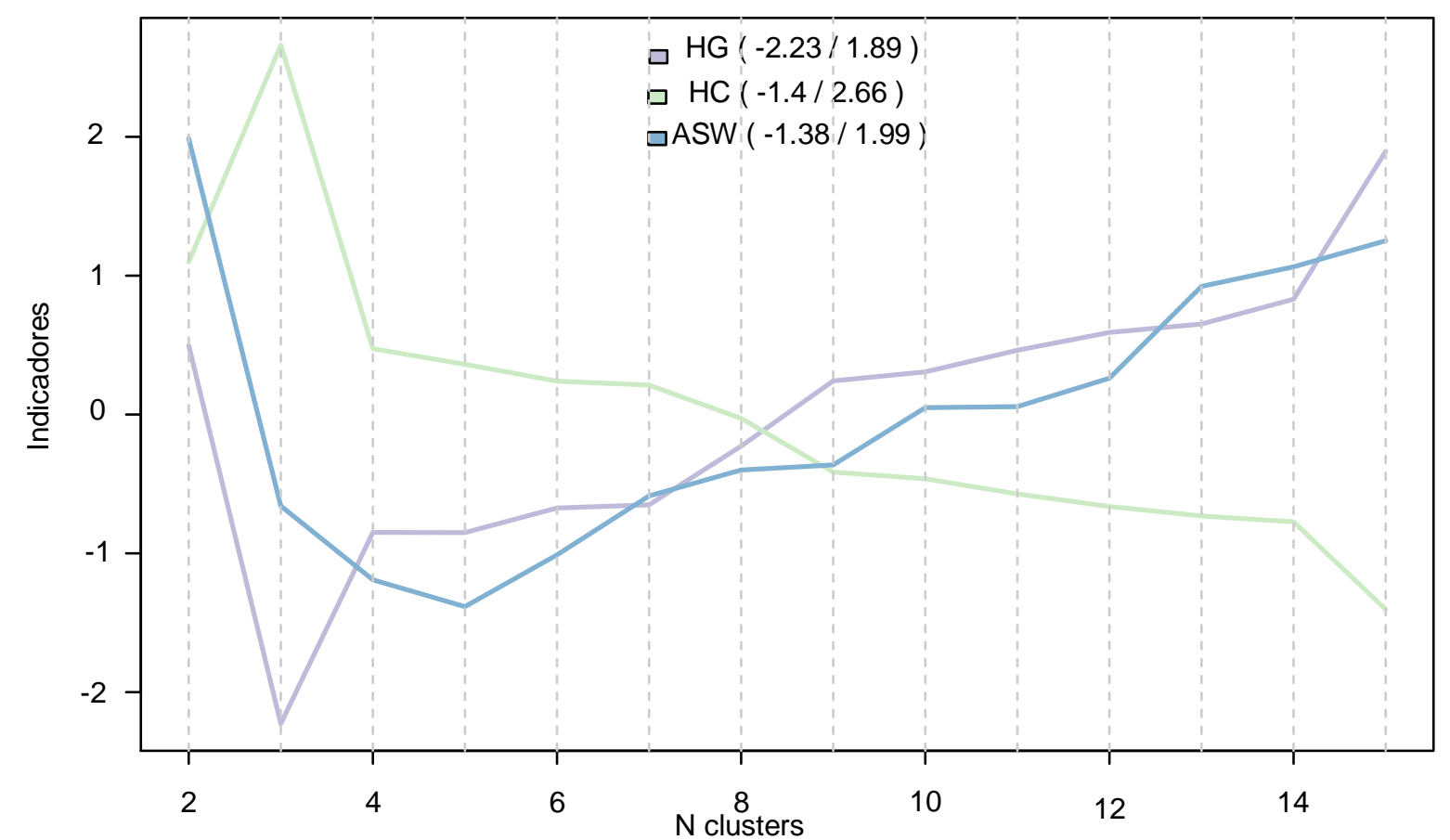

Fig. 6: Distribución de criterios de optimalidad para proporción 50:50

En este caso se escogieron diez clusters, teniendo en cuenta que es el menor número de grupos que cumplen las condiciones de optimalidad simultáneamente. En la figura 7, se presenta esta nueva forma de agrupamiento. En esta propuesta, en la cual se contempla un valor del $50 \%$ para vecindad muscular y un $50 \%$ para terneza, se puede apreciar que los cortes van tomando una posición un poco más cercana a la realidad en cuanto a la práctica de desposte se refiere, sin embargo, aunque varios cortes son posibles (Cluster 1, p.e,), la mayoría no lo son. Haciendo un desglose por continuidad, aparecerían 16 cortes reales; número bastante elevado. Lo anterior indica que lograr una uniformidad completa en un corte en cuanto a terneza y continuidad de sus músculos se refiere no es completamente viable.

Cabe resaltar que este tipo de estudio implementado para la presente investigación muestra un componente innovador, debido a que, en estudios similares relacionados con los cortes de carne de los diferentes sistemas de clasificación de canales, no se encuentran evidencias del agrupamiento a partir de una ponderación estadística teniendo en cuenta valores como la terneza y la vecindad anatómica. Para la industria cárnica, representará un apoyo innovador importante no sólo para la clasificación de canales y cortes, sino para el análisis de la calidad de la carne. 


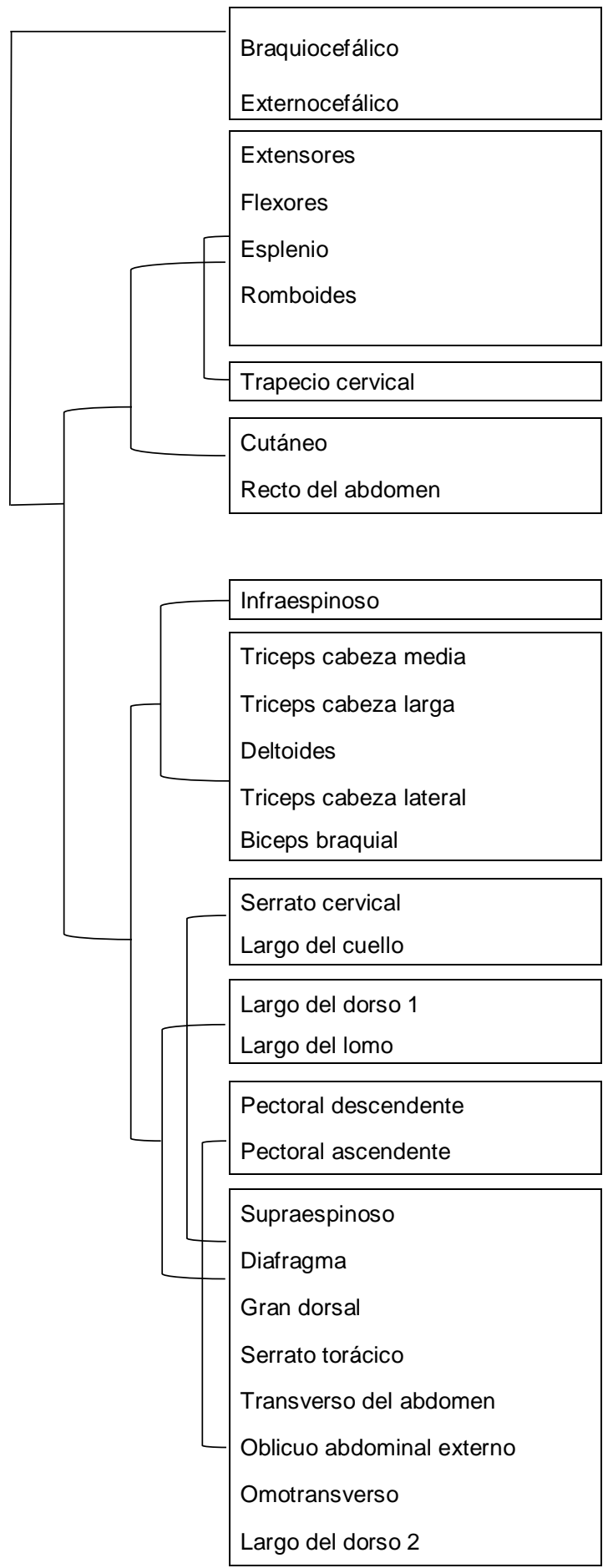

Fig. 7: Grupo de músculos según ponderación vecindad: terneza 50:50.

\section{CONCLUSIONES}

La terneza en los músculos del tren delantero de la canal bovina medida por WBS no varía significativamente entre canales de clasificación 3, 4 y 5 estrellas, pero si dentro de la misma canal. Históricamente los músculos de la postura se han catalogado como más tiernos y de mejor calidad, sin embargo, se evidenció que los principales músculos que conforman el brazo resultaron ser más tiernos que los músculos que conforman el lomo. Por lo tanto, con una mejor comprensión de la terneza de los principales músculos que conforman el tren delantero del bovino se puede hacer un mejor uso de aquellos músculos infrautilizados para desarrollar nuevos productos o cortes. 
El apoyo en medios estadísticos para la clasificación de cortes y canales no sólo de bovinos, sino de porcinos, ovinos y demás animales de abasto, puede representar un avance importante para la industria cárnica, debido a que se pueden tener para dicho proceso, la ponderación y el agrupamiento de diferentes músculos en posición anatómica similar como referentes y correlacionarlos con valores como la terneza, la vecindad, el $\mathrm{pH}$, la composición bromatológica, entre otros.

\section{AGRADECIMIENTOS}

Los autores expresan su agradecimiento al Ministerio de Ciencia Tecnología e Innovación Colombiano (Minciencias) y a la Secretaría de Agricultura del Departamento de Antioquia, Colombia, por su apoyo financiero para la ejecución de la investigación Bases Científicas y Tecnológicas para la Puesta en Marcha de un Sello de Calidad de Carne Bovina en la Región del Urabá Antioqueño. Contrato N 80740-010-2019. Proyecto $\mathrm{N}^{\circ} 63343$ y a José A. Cardona H., por su apoyo en la estructuración formal de este artículo.

\section{REFERENCIAS}

Anderson, M. J., Lonergan, S. M., y otros cuatro autores, Profile of biochemical traits influencing tenderness of muscles from the beef round, https://doi.org/10.1016/j.meatsci.2012.01.022, Meat Science, 91(3), 247-254 (2012)

Apaoblaza, A., Galaz, A., y otros cuatro autores, Glycolytic potential and activity of adenosine monophosphate kinase (AMPK), glycogen phosphorylase (GP) and glycogen debranching enzyme (GDE) in steer carcasses with normal (<5.8) or high (> 5.9) $24 \mathrm{H} \mathrm{pH}$ determined in m. longissimus dorsi, https://doi.org/10.1016/j.meatsci.2014.11.008, Meat Science, 101, 83-89 (2015)

Braña V. D., Ramírez R. E., y otros siete autores, Manual de análisis de calidad en muestras de carne, Folleto Técnico No 11, Queretáro, México (2011)

Bourne, M. C., Kenny, J. F., y Barnard, J., Computer-assisted readout of data from texture profile analysis curves 1 , https://doi.org/10.1111/j.1745-4603.1978.tb01219.x, Journal of Texture Studies, 9(4), 481-494 (1978)

Choi, Y. M., Lee, S. H., y otros cinco autores, Protein solubility is related to myosin isoforms, muscle fiber types, meat quality traits, and postmortem protein changes in porcine longissimus dorsi muscle, https://doi.org/10.1016/j.livsci.2009.09.009, Livestock Science, 127(2-3), 183-191 (2010)

Chun, C. K. Y., Wu, W., y otros cinco autores, A preliminary investigation of the contribution of different tenderness factors to beef loin, tri-tip and heel tenderness, https://doi.org/10.1016/j.meatsci.2020.108247, Meat Science, 170, 108-247 (2020)

Costa, P., Simões, J. A., y otros seis autores, Repercussions of growth path on carcass characteristics, meat colour and shear force in alentejana bulls, https://doi.org/10.1017/S1751731115000427, Animal, 9(8), 1414-1422 (2015)

Della Rosa, M. M., Mazzucco, J. P., y Aello, M. S., Relación de la dieta con el color y la terneza de la carne vacuna. Archivos de Zootecnia, 66(255), 459-467 (2017)

González, A., Alvis, A., y Arrázola, G., Efecto del recubrimiento comestible en las propiedades de trozos de batata (Ipomoea batatas lam) fritos por inmersión: parte 1: textura, doi:10.4067/S0718-07642015000100011, Información Tecnológica, 26(1), 95-102 (2015)

Hubert, L., y Schultz, J., Quadratic assignment as a general data analysis strategy, doi: 10.1111/j.2044-

8317.1976.tb00714, British Journal of Mathematical and Statistical Psychology, 29(2),190-241 (1976)

Listrat, A., Gagaoua, M., y otros ocho autores, Are there consistent relationships between major connective tissue components, intramuscular fat content and muscle fibre types in cattle muscle?, https://doi.org/10.1017/S1751731119003422, Animal, 14(6), 1204-1212 (2020)

Motter, M. M., Corva, P. M., y Soria, L. A., Expression of calpastatin isoforms in three skeletal muscles of angus steers and their association with fiber type composition and proteolytic potential, https://doi.org/10.1016/j.meatsci.2020.108267, Meat Science, 171, 108-267 (2021)

Motter, M., Corva, P., y otros cuatro autores, Tipo de fibra y proteólisis en músculos de novillos angus, Revista Argentina de Producción Animal, 38(1), 15-21 (2018)

Nair, M. N., Canto, A. C., Rentfrow, G., y Suman, S.P., Muscle-specific effect of aging on beef tenderness, https://doi.org/10.1016/j.Iwt.2018.10.038, LWT, 100, 250-252 (2019)

NTC 4271, Sistema ICTA de clasificación de canales y cortes de carne bovina, 8-14, Bogotá, Colombia (1996)

Popesko, P., Atlas de anatomía topográfica de los animales domésticos, 2a ed, 8-208, Masson, Barcelona, España, (1998)

Purchas, R. W., y Zou, M., Composition and quality differences between the longissimus and infraspinatus Muscles for several groups of pasture-finished cattle, https://doi.org/10.1016/j.meatsci.2008.01.013, Meat Science, 80(2), 470-479 (2008)

R Core Team, R: A language and environment for statistical computing, R Foundation for Statistical (2021) 
Rousseeuw, P.J., Silhouettes: a graphical aid to the interpretation and validation of cluster analisis, https://doi.org/10.1016/0377-0427(87)90125-7, Journal of Computational and Applied Mathematics, 20, 53-65 (1987)

Searle, S.R., Speed, F.M., y Milliken, G.A., Population marginal means in the linear model: an alternative to least Squares Means, https://doi.org/10.1080 / 00031305.1980.10483031, The American Statistician, 34 (4), 216-221 (1980)

Sullivan, G. A., y Calkins, C. R., Ranking beef Muscles for warner-bratzler shear force and trained sensory panel ratings, doi:10.1111/j.1745-4557.2011.00386.x, Nebraska Beef Cattle Reports, 90 (2007)

Wright, S. A., Ramos, P., y otros siete autores, Brahman genetics influence muscle fiber properties, protein degradation, and tenderness an Angus-Brahman multibreed herd, https://doi.org/10.1016/j. meatsci.2017.09.006, Meat Science, 135, 84-93 (2018)

Wu, G., Farouk, M. M., Clerens, S., y Rosenvold, K., Effect of beef ultimate pH and large structural protein changes with aging on meat tenderness, https://doi.org/10.1016/j.meatsci.2014.06.010, Meat Science, 98(4), 637-645 (2014)

Yeh, Y., Omaye, S. T., y otros tres autores, Evaluation of palatability and muscle composition of novel value-added beef cuts, https://doi.org/10.1016/j.meatsci.2017.08.026, Meat Science, 135, 79-83 (2018) 